\title{
Efficient Orthonormality Testing for Synthesis with Pass-Transistor Selectors
}

\author{
Michel Berkelaar,michel@es.ele.tue.nl \\ Eindhoven University of Technology, P.O. Box 513, 5600 MB Eindhoven, the Netherlands
}

Lukas P.P.P. van Ginneken, lukas@synopsys.com

Synopsys Inc., 700 East Middlefield Road, Mountain View, CA 94043

\begin{abstract}
This paper presents the mapping problem for pass transistor selector mapping, which has not been addressed before. Pass transistor synthesis is potentially important for semi-or full-custom design techniques, which are increasingly attracting attention. Pass transistors have the advantage that fewer transistors are needed, and that circuits with high fanin and small delay can be constructed. Technology mapping approaches in the existing literature cannot handle these selectors, due to the restriction of 1-hot encoding of the control signals. We present a new algorithm to address this problem, which is based on the novel idea of a general Boolean Oracle. Our oracle is based on ATPG techniques, and compared to BDDs, the oracle has the advantage that failure to complete only affects optimization locally, and does not hinder optimization elsewhere in the logic. A limitation of BDDs is that it is difficult to complete the algorithm if a BDD grows too large. The experimental results show up to $82 \%$ improvement in transistor count for the MCNC combinatorial multi-level examples.
\end{abstract}

\section{Introduction}

In this paper a selector gate is a gate with $n$ pairs of inputs. Each input pair has a data and a control input. The output of the selector gate is defined only for the case that exactly one of the $n$ control inputs is 1 . In this paper we call a set of signals in which exactly one is 1 for any input vector orthonormal. A formal definition can be found in section 2. In case of orthonormal control inputs, the (single) output of the selector gate will reflect the value of the data input associated with the control input that is 1 . A related type of gate is the multiplexer gate. Multiplexers have data and control inputs too, but they do not have a one-to-one correspondence of data and control inputs. In most cases multiplexers have fewer control inputs than data inputs. Some kind of decoding of the values on the control inputs determines which data input controls the multiplexer output. See Figure 1 for an example of a 4 input selector and a 4 input multiplexer with 2 control inputs. In this paper we will only discuss the problem of using selectors to implement logic.

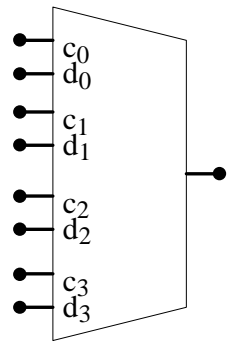

4 input selector

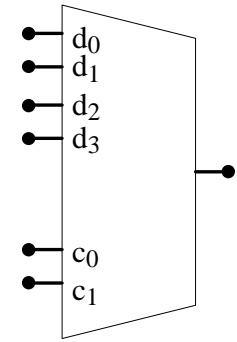

4 input multiplexer
Figure 1: difference between selector and multiplexer

The problem of using the selectors which might be available in a standard cell library during technology mapping is a fundamental one. All technology mappers that the authors are aware of require that all standard cell library elements without memory can be described by a single (possibly multi-output) Boolean function. This Boolean function is internally represented by, for example, a network of 2-input nands and inverters (e.g. the MIS mapper [DET87]), or a BDD (e.g. CERES [MAI90]), or even a truth table (e.g. the BooleDozer mapper). This function is supposed to hold for all possible input values. Selectors also perform a Boolean function from inputs to output (a simple and-or function), but this function is only defined for those cases where the control inputs are orthonormal. The actual behavior for other control input values of a selector gate is implementation dependent. The pass transistor implementation of Figure 2 will have a high output impedance when all control inputs are 0 , and produce some intermediate value with a high short circuit current flowing when 2 control signals are 1 with conflicting corresponding data input signals. In practical implementations it might be necessary to add an inverter or buffer to the output of a pass transistor circuit to restore the logic values, but it still remains a very efficient implementation. An introduction to pass-transistor logic can be found in [WHI81].

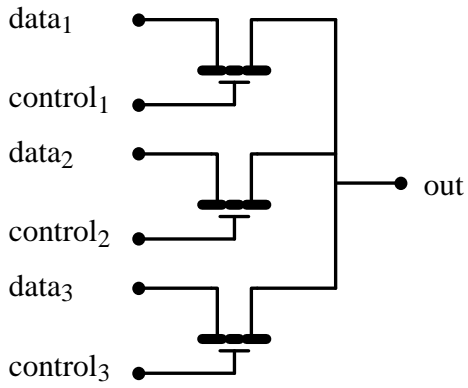

Figure 2: 3 input pass transistor selector

All of this implies that we can only map parts of a circuit to a selector after we have made sure that the control signals will be orthonormal. This function cannot be performed by a 'normal' technology mapper. Yet, selectors can be very efficient implementations for some pieces of logic, and are, therefore, present in some standard cell libraries.

This paper will describe an approach to identify those parts of circuits that can be mapped to selectors.

This paper is the first one on the topic of mapping to selectors that the authors are aware of. A lot of attention has been given to mapping circuits to multiplexer based FPGAs (e.g. [ZHU93] [LAN93]), but the problem of the requirement of orthogonal control signals does not occur here. Furthermore, we are not trying to implement a circuit by means of selectors only. We only want to replace the parts where this is "natural" and does not require the introduction of extra logic nor substantial restructuring of the logic. This paper is organized as follows. Section 2 introduces the concept of a Boolean Oracle. We use this oracle to answer questions about the orthonormality and orthogonality of signals. In section 
3 of this paper we describe how we identify parts of circuits that can be replaced by selectors. In section 4 we show how some of the selectors we have identified in section 3 can be merged into fewer but larger selectors. Section 5 presents results on selectors found in the set of MCNC combinatorial multi-level examples. Section 6 contains the conclusions.

\section{The Boolean Oracle}

To be able to use selectors to implement parts of logical circuits, we need to be able to answer questions about the orthogonality and orthonormality of sets of signals. In the paper we assume that we have a Boolean Oracle to answer our questions. To define what kind of questions we expect the Boolean Oracle to answer, we need some definitions. The word circuit will refer to a combinatorial Boolean circuit consisting of gates connected by signals.

Definition 1: The set $\varphi$ is the set of all signals in a circuit.

Definition 2: The set $\mathcal{N}$ is the set of all nands in a circuit.

In the following definitions we use the notation $\varphi^{*}$ to indicate the power set of $\varphi$.

Definition 3: The function Fanin : $\mathcal{N} \rightarrow \varphi^{*}$ gives the set of input signals of a nand.

Definition 4: $\mathscr{V}$ is the set of all possible primary input vectors for a circuit. For a circuit with $n$ primary inputs without input don't cares, $|\mathcal{V}|=2^{n}$. If there are don't care conditions on the inputs, $|\mathcal{V}|<2^{n}$.

We will use statements like $\exists_{v \in q}$ formula or $\forall_{v \in q}$ formula, even though $v$ might not appear in formula. But, as we limit ourselves to combinatorial logic circuits, other signals values $s$ in the circuit, which do appear in formula, depend directly on $v$, formally requiring a notation like $s(v)$. Where this does not lead to ambiguity, we will simply write $S$.

Definition 5: A set of signals $S$ in a Boolean network is called orthogonal when

$\forall_{v \in \mathcal{V}}\left(\forall_{s \in S}\left(s=1 \Rightarrow \forall_{s^{\prime} \in S \mid\{s\}}\left(s^{\prime}=0\right)\right)\right)$

or: at most one of the signals is 1 for any input vector applied to the circuit.

Definition 6: A set of signals $S$ in a Boolean network is called orthonormal when it is orthogonal and

$\forall_{v \in \mathcal{V}}\left(\exists_{s \in S} s=1\right)$

or: exactly one of the signals is 1 for any input vector applied to the circuit.

In the rest of the paper, $\wedge$ will indicate the Boolean and operation. Definition 7: The function All_1: $\varphi^{*} \rightarrow\{0,1\}$ can be defined for $S \subseteq \varphi$ by:

All_1 $1(S)= \begin{cases}0 & \forall_{v \in \mathcal{V}}\left(\bigwedge_{s \in S} S=0\right) \\ 1 & \exists_{v \in \mathcal{V}}\left(\bigwedge_{s \in S} s=1\right)\end{cases}$

All_1(S) indicates whether or not the signals $S \in S$ can all be 1 for some input vector.

Definition 8: The function All_0: $\varphi^{*} \rightarrow\{0,1\}$ can be defined for $S \subseteq 9$ by:

$A$ II_O $(S)= \begin{cases}0 & \forall_{v \in q}\left(\bigwedge_{s \in S} \bar{s}=0\right) \\ 1 & \exists_{v \in q}\left(\bigwedge_{s \in S} \bar{s}=1\right)\end{cases}$

$A / l 0(S)$ indicates whether or not the signals $S \in S$ can all be 0 for some input vector.
A Boolean Oracle can calculate the value of $A / l 1$ or $A / l$ 0. It could be implemented in several ways:

1. By exhaustive simulation of the circuit. This might be very time consuming, and is not a realistic option for circuits with many primary inputs.

2. By using BDDs. BDDs are known to grow exponentially for certain types of circuits, but behave reasonably for many practical circuits.

3. By using a test pattern generator.

We have opted for the last implementation. We use the package basically by setting the signals in a set $S \subseteq \varphi$ to fixed 0 or 1 values, and then ask the test pattern package to come up with an input pattern that will generate these internal signal values. This uses only the justification capability of the test pattern package. There are 3 possible answers:

1. If an input pattern can be found, we have set the signals to valid values.

2. If no input pattern can be found because of inconsistencies, we have set the signals to values which can never occur.

3. The test pattern package cannot answer the question conclusively because it would take too much time.

The test pattern package we use is TGFS [KUN90] [KUN92], which is part of the IBM BooleDozer ${ }^{1}$ logic synthesis system. The speed of this test pattern package allows us to consult the Boolean Oracle many times.

A very important reason for choosing to implement the Boolean Oracle by using TGFS is the graceful way in which it fails, as compared to BDDs. If we had decided to use BDDs, we would not have been able to reason about circuits for which the BDD representation blows up. The process will run out of memory, or hit a memory limit specified to the BDD package, and we will fail to produce results for that circuit. A test generator will not run out of memory, but it might use a lot of time. However, TGFS allows one to specify the amount of effort that should be spent on a specific question. This means that the oracle may sometimes respond to a question with the answer "don't know". In the context of this paper, this means that sometimes we cannot be sure whether a selector can be used, and have to decide not to do it. But in other places in the same circuit we may still be able to decide. For the MCNC benchmarks used in the results section of this paper, the oracle was always able to give a decisive answer. Any BDD packages would have had a hard time with the multiplier of circuit C6288, and BDD packages without dynamic variable ordering would have found other circuits difficult too [RUD93].

\section{Identifying selectors}

We will look for selectors in the logic in three basic steps:

1. Find parts of the circuits where the basic and-or function of the selector is performed. These parts are called candidates.

2. In each of the candidates, try to identify sets of signals which are orthogonal, and, therefore, might serve as control inputs of a selector.

3. Finally, check if the sets of control signals identified in the previous step are orthonormal. If the answer is yes, we have found a complete selector. If the answer is no, the control signals are only orthogonal and the selector is incomplete, but this can be fixed by adding another input to it.

1. BooleDozer is a trademark of IBM Corp. 


\section{Finding candidates}

We start by looking for parts of the circuit that perform an and-or function. We do this by transforming the circuit to nands only, where nand-inverter-nand constructions without intermediate fanout are merged into a larger nand. Every two-level tree of nands now performs an and-or function, and is a candidate for (partial) replacement with a selector. The ideal form of this transformation, where the entire candidate can be replaced, is pictured in Figure 3. We will call the nands in the leftmost, input part of a

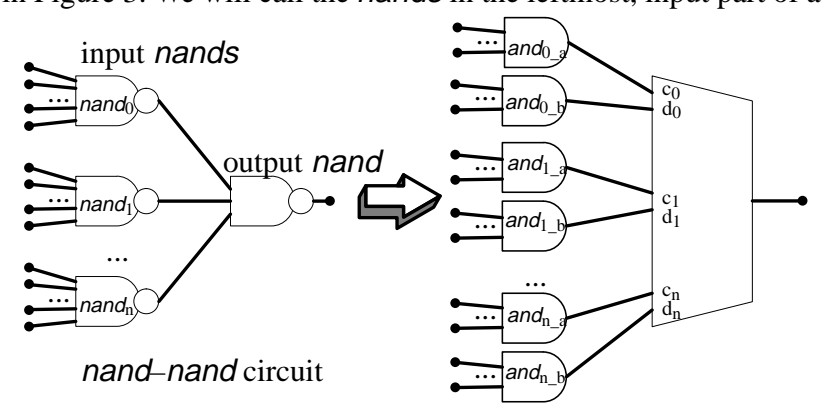

Figure 3: From nand tree to selector circuit

two-level nand tree the input nands. The nand generating the output is called the output nand. For every input nand, we have to find a partition of its input signals into a control and a data subset. The logical and of the control subset will form one control input of the selector, and the logical and of the data subset the data input. Another version of this transformation is when we only replace a part of the $n$ input nands by a selector. In that case we have to keep a smaller output nand around, and invert the output of the selector (or use an inverting selector). This is pictured in Figure 4.

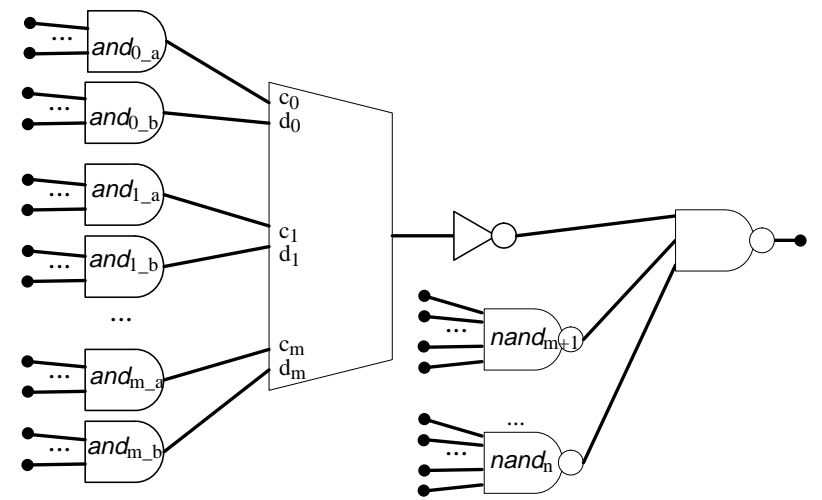

Figure 4: partial replacement of nand tree by selector circuit

\section{Finding sets of control signals in candidates}

The task we face is to find parts of two-level nand-nand candidates which can be implemented correctly by a selector. For each such selector we have to figure out which of the input signals to the input nands of a candidate form the control part of this select bit, and which signals the data part. We answer this question first with respect to pairs of input nands of candidates. For each such pair we are going to find all correct sets of signals which could be used as the control signal if these two nands were absorbed into a selector. The data signals are of course implicitly the remaining input nand inputs. If no data input remains, the constant value 1 is used. The sets of input signals which can be used as control signals for an input nand pair are called matches and form a match matrix for every candidate. Matches are formally introduced by the following definition:

Definition 9: A match $m$ of a pair $\left(\right.$ nand $_{i}$, nand ${ }_{j}$ ) of input nands of a single candidate is a pair of non-empty sets of signals: $m=\left\{S_{i}, S_{j}\right\}, S_{i} \subseteq$ Fanin $\left(\right.$ nand $\left._{i}\right), S_{j} \subseteq$ Fanin(nand $\left.)_{j}\right)$ $S_{i} \neq \emptyset, S_{j} \neq \emptyset$, All $\_1\left(S_{i} \cup S_{j}\right)=0$

Theorem 1: For a match $m=\left\{S_{i}, S_{j}\right\}$, let $c_{1}=\bigwedge_{s \in S_{i}} s$, the logical and of the signals $s \in S_{i}$, and $c_{2}=\bigwedge_{s \in S_{j}} s$, the logical and of the signals $s \in S_{j}$, then $c_{1}$ and $c_{2}$ are orthogonal signals.

Proof: We first prove the (normal) case when $S_{i} \nsubseteq S_{j}$ and $S_{j} \nsubseteq S_{i}$. Because of symmetry we only need to prove that $c_{1}=1 \Rightarrow c_{2}=0$. From $c_{1}=1$ it follows that $\exists_{v \in \mathcal{V}}\left(\bigwedge_{s \in S_{i}} s=1\right)$. Because there is a match $\left\{S_{i}, S_{j}\right\}$, it follows that $\forall v \in \mathcal{v}\left(\bigwedge_{s \in S_{j} \cup S_{j}} s=\bigwedge_{s \in S_{i}} s \wedge \bigwedge_{s \in S_{j}} s=0\right)$. This can only be true if $\quad \forall v \in v\left(c_{1}=1 \Rightarrow \bigwedge_{s \in S_{j}} s=0\right)$, from which $c_{1}=1 \Rightarrow c_{2}=0$ follows directly.

In the less likely case that $S_{i} \subseteq S_{j}$ or $S_{j} \subseteq S_{i}$, we get the following. Again, because of symmetry we only treat one case, $S_{i} \supseteq S_{j}$.

$\left\{S_{i}, S_{j}\right\}$ is a match, so $\forall_{v \in \mathcal{v}}\left(\bigwedge_{s \in S_{j}, S_{j}} s=0\right)$. Also, $S_{i} \supseteq S_{j} \Rightarrow S_{j} \mid S_{i}=\emptyset$. We can now write $\forall v \in v\left(\bigwedge_{s \in S_{j}, S_{j}} s=\bigwedge_{s \in S_{i}} s \wedge \bigwedge_{s \in S_{j} \mid s_{i}} s=\bigwedge_{s \in S_{i}} s \wedge 1=0\right)$.

Therefore, $\forall_{v \in q} c_{1}=0$. A pair of signals, of which one is always 0 , is orthogonal.

If we think of the inputs of an input nand as being ordered, we can represent a match between an input nand with $n$ inputs and an input nand with $m$ inputs as a pair of two bit vectors of length $n$ and $m$ respectively, where a 1 in the bit vector corresponds to an input signal being part of the match, and a 0 to a choice as data signal. An example of a match matrix is given in Figure 5. All matches between input nand 1 and input nand 2 are listed in match matrix entry $(2,1)$. Of course, the entry in $(1,2)$ would be identical, we don't need to store it. The diagonal does not carry any values either. But we do have to store $n / 2 \times(n-1)$ entries if the output nand has a fanin of $n$.

Theorem 2: If $m=\left\{S_{i}, S_{j}\right\}$ is a match, then

$m^{\prime}=\left\{S^{\prime}{ }_{i}, S^{\prime}{ }_{j}\right\}, \operatorname{Fanin}\left(\right.$ nand $\left._{i}\right) \supseteq S_{i}{ }_{i} \supseteq S_{i}$,

Fanin $\left(\right.$ nand $\left._{j}\right) \supseteq S_{j}^{\prime} \supseteq S_{j}$ is a match too. If $S^{\prime}{ }_{i} \supset S_{i} \vee S^{\prime} \supset S_{j}$, we call these matches implied matches.

Proof: For $m$ holds: All_1 $\left(S_{i} \cup S_{j}\right)=0$. This implies

$\forall v \in \mathcal{v}\left(\bigwedge_{s \in S_{j}, S_{j}} s=0\right)$. We need to proof that 
$\forall v \in\left(\bigwedge_{s \in S^{\prime}, \nu S_{j}^{\prime}} s=0\right)$

We know that $S^{\prime}{ }_{i} \cup S^{\prime}{ }_{j} \supseteq S_{i} \cup S_{j}$ We will define the difference set $S_{\text {diff }}=S_{i}{ }_{i} \cup S^{\prime}{ }_{j} \mid\left(S_{i} \cup S_{j}\right)$. Now it is easy to see that

$\forall_{v \in V}\left(\bigwedge_{s \in S_{j}, S_{j}, S_{\text {diff }}} s=\bigwedge_{s \in S_{j}, S_{j}} s \wedge \bigwedge_{s \in S_{\text {diff }}} s=0 \wedge \bigwedge_{s \in S_{\text {diff }}} s\right)$

All matches with a gray background in Figure 5 are implied

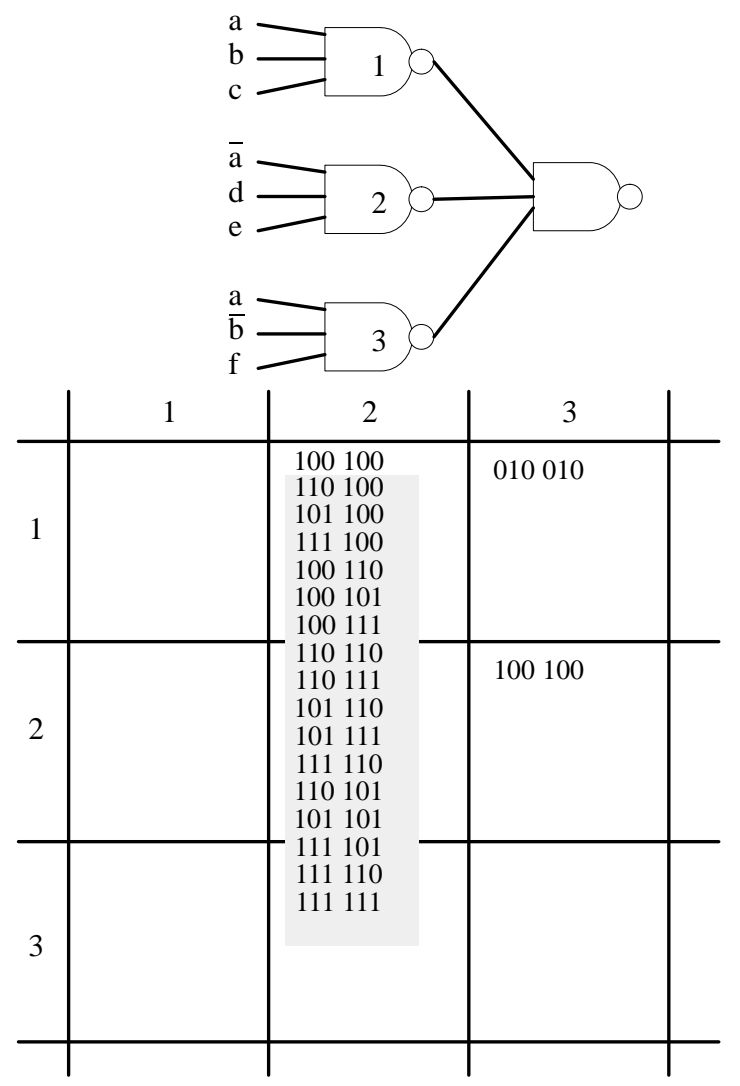

Figure 5: Match matrix

matches. We only included them for entry $(2,1)$, for the other entries they can be constructed in a similar way. This observation leads to the following two rules:

1. When looking for matches, start looking for matches with as few signals as possible. This way we will find all matches that could not possibly be implied by another match first, and can easily skip testing of implied matches.

2. Store only the matches with minimal number of signals. All matches that are implied from this one can easily be derived and need not be stored.

There are two other ways we speed up the process of finding matches. The first property we exploit is given by the following theorem, which is in a sense the dual of Theorem 2;
Theorem 3: If $\left\{S_{i}, S_{j}\right\}$ is not a match, then $\left\{S^{\prime}, S^{\prime}{ }_{j}\right\}, \emptyset \subset S^{\prime} \subseteq S_{i}, \emptyset \subset S^{\prime} \subseteq S_{j}$ is a not a match either.

Proof: If $\left\{S_{i}, S_{j}\right\}$ is not a match, it follows that $\exists_{v \in \mathcal{V}}\left(\bigwedge_{s \in S_{j}, S_{j}} s=1\right) \Rightarrow \exists_{v \in \mathcal{V}}\left(\forall_{s \in S_{j}, S_{j}} s=1\right)$. Therefore, for $\quad$ all $\quad\left\{S^{\prime}{ }_{i}, S^{\prime}{ }_{j}\right\}, \emptyset \subset S^{\prime}{ }_{i} \subseteq S_{i}, \emptyset \subset S^{\prime} \subseteq S_{j}$ holds $\exists_{v \in \mathcal{V}}\left(\bigwedge_{s \in S_{j}^{\prime}, S_{j}^{\prime}{ }_{j}} s=1\right)$, and it is not a match.

From Theorem 3 it immediately follows that:

Corollary 4: For any two input nands nand, nand $_{j}$ of a single

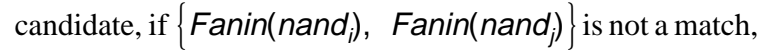
then there are no matches for this input nand pair.

Thus, the first test we do for any input nand pair is to ask the oracle

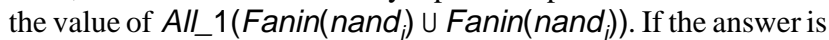
1 , there are no matches for this pair. This single question to the Boolean Oracle can avoid many other questions to identify the matches.

The second thing we do is identifying counter examples for matches by performing random simulations. In each candidates nomatch matrix we store nomatches.

Definition 10: A nomatch nom of a pair ( nand $_{i}$, nand $_{j}$ ) of input nands of a single candidate is a pair of non-empty sets of signals:

$$
\text { nom }=\left\{S_{i}, S_{j}\right\}, S_{i} \subseteq \operatorname{Fanin}\left(\text { nand }_{i}\right)
$$

$S_{j} \subseteq \operatorname{Fanin}\left(\right.$ nand $\left._{j}\right), S_{j} \neq \emptyset, \quad S_{j} \neq \emptyset A l l \_1\left(S_{j} \cup S_{j}\right)=1$

During random simulation of the circuit after identifying the candidates, we observe the input pins of input nands of candidates and store the nomatches. With this set of nomatches and Theorem 3 we can identify many pairs $\left\{S_{i}, S_{j}\right\}$ as definitely not being matches, and we do not have to bother the oracle for the decision.

We can now write down the algorithm to find the matches:

Algorithm 1: find matches

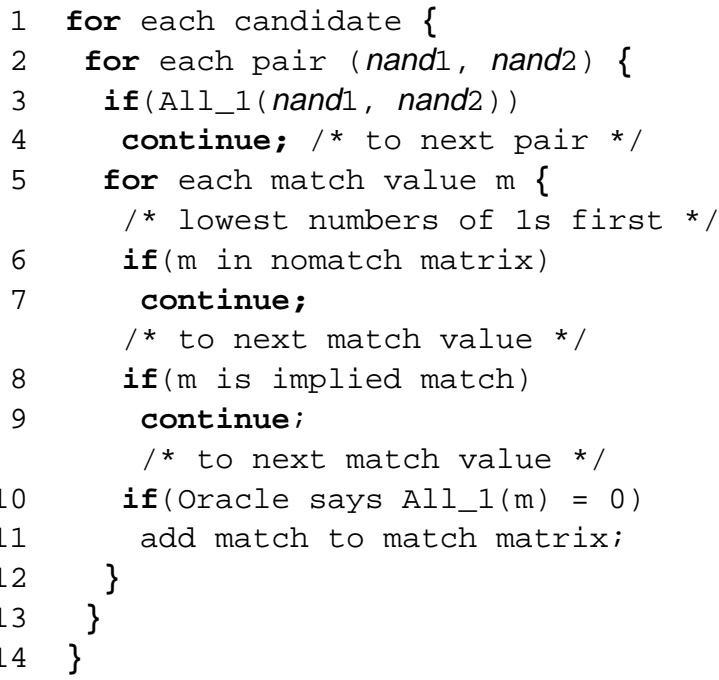

The run time of algorithm 1 is quadratic in the number of input nands of a candidate. If the test on line 3 fails, for a pair with a $n$ 
and a $m$ input nand, we actually must check all $\left(2^{n}-1\right) \times\left(2^{m}-1\right)$ different possible match values. This is a very bad worst case time complexity, but in practical situations this algorithm turns out be fast enough even for large circuits. In our implementation the designer can limit the fanin of output nands and input nands of candidates to be considered to limit the run time if needed. By default, we do not consider candidates with more than 50 input nands, nor input nands with more than 20 inputs.

Table 1 shows how efficiently the heuristics in algorithm 1 limit the number of times we have to ask the Boolean Oracle if a match is valid. We have chosen the largest 10 circuits (in terms of number of connections in the circuit) from the MCNC combinatorial multilevel benchmark circuits in which we find selectors (see the Results section and Table 2) to show this. The size of a circuit is not directly related to run time for our algorithms, but this subset contains the 2 examples consuming the most CPU time. Column 'total tests performed' list the number of calls to the Boolean Oracle. Column 'total tests skipped' lists the number of times we decided we knew the answer before asking the oracle. Column 'initial pair test skip' shows the number of calls to the oracle we did not make because the test on line 3 of Algorithm 1 succeeded. Column 'nomatch skip' shows the number of times the test on line 6 succeeded. Column 'implied match skip' shows the number of times the test on line 8 succeeded, indicating a match was implied by a previously found match. This number is also equal to the number of matches that need not be stored. The actual number of matches we did have to store is listed in column 'matches stored'. The number of nomatches we found and stored during random simulation of the circuit with 1024 input vectors is listed in column "nomatches stored'. The last column lists the CPU time in seconds needed on an IBM RS6000/370 (approximately 60 MIPS) to run the match finding algorithm. Table 1 shows that we succeeded in finding all matches, while making actual calls to the Boolean Oracle only in a small fraction of the cases. The number of matches stored for each example indicates the theoretical lower limit for the number of calls to the Boolean Oracle that need to be made. A real match can only be found by an All_1 test, as all shortcuts we use lead to a negative conclusion. All three tests in algorithm 1 contribute significantly to this result. The fact that we have chosen not to store implied matches also limited our storage needs for matches to a very acceptable number.

Table 1: Efficiency of match finding

\begin{tabular}{|l|r|r|r|r|r|r|r|r|}
\hline name & $\begin{array}{l}\text { total } \\
\text { tests } \\
\text { done }\end{array}$ & $\begin{array}{l}\text { total } \\
\text { tests } \\
\text { skip }\end{array}$ & $\begin{array}{l}\text { initial } \\
\text { pair } \\
\text { test } \\
\text { skip }\end{array}$ & $\begin{array}{l}\text { no- } \\
\text { match } \\
\text { skip }\end{array}$ & $\begin{array}{l}\text { implied } \\
\text { match } \\
\text { skip }\end{array}$ & $\begin{array}{l}\text { match } \\
\text { es } \\
\text { stored }\end{array}$ & $\begin{array}{l}\text { nomat } \\
\text { ches } \\
\text { stored }\end{array}$ & $\begin{array}{l}\text { cpu } \\
\text { (s) }\end{array}$ \\
\hline \hline C5310 & 1473 & 11184 & 84 & 8242 & 2858 & 692 & 1776 & 14 \\
\hline C7552 & 2418 & 31459 & 23004 & 5893 & 2562 & 554 & 1541 & 44 \\
\hline dalu & 873 & 18794 & 363 & 9914 & 8472 & 383 & 1319 & 16 \\
\hline des & 6154 & 800117 & 9524 & 523347 & 267246 & 3006 & 15749 & 124 \\
\hline frg2 & 6679 & 115089 & 6580 & 84948 & 23561 & 195 & 1699 & 40 \\
\hline i10 & 1527 & $2.3 \mathrm{E} 6$ & $2.3 \mathrm{E} 6$ & 10491 & 4711 & 446 & 1751 & 28 \\
\hline i8 & 2934 & 83168 & 2180 & 61029 & 19959 & 1084 & 5699 & 38 \\
\hline pair & 1275 & 5464 & 3588 & 1560 & 316 & 279 & 1996 & 21 \\
\hline x1 & 74543 & $2.6 \mathrm{E} 9$ & $2.6 \mathrm{E} 9$ & $3.7 \mathrm{E} 6$ & $1.3 \mathrm{E} 6$ & 235 & 60707 & 447 \\
\hline x3 & 1274 & 28092 & 13332 & 11356 & 3404 & 245 & 1472 & 15 \\
\hline
\end{tabular}

\section{From matches to selectors}

We now know if and how pairs of input nands could be part of a selector. But, of course, we want the selectors to absorb as many of the input nands of a candidate as possible. In many respects this is not a simple task. It would be best if we could replace the entire candidate by a single selector. But in many cases this is not possible. In general we can replace a candidate by a number of selectors and some of the original input nands, much like in Figure 4. In many cases, several different configurations will be possible, some of which could be superior in some respect to others. We currently use a heuristic algorithm to find a set of 'good' selectors for every candidate. It is based on the concept of growing a selector consisting of 2 or more input nands by adding one more input nand to it. A selector is represented for these purposes by a set of input nands, each with a known partition of inputs into control and data inputs. A trivial starting point is of course taking a pair of input nands for which a match, specifying the partitions can be found in the match matrix. We can try to grow this configuration by adding one more input nand to it. This is only possible if the new input nand has a non-empty set of matches with the nands already chosen. In that case we have to figure out how the control subsets for construction of the larger selector should be.

As an example. let's consider the circuit of Figure 5. Remember that the match matrix does not actually have the gray entries. If we start with pair $n a n d_{0}$ and $n a n d_{1}$, we get the following selector:

\begin{tabular}{|c|c|}
\hline nand $_{1}$ & nand $_{2}$ \\
100 & 100 \\
\hline
\end{tabular}

If we try to add the third nand, the match matrix tells us that we have the following choices for the control inputs:

\begin{tabular}{|c|c|c|}
\hline nand $_{1}$ & nand $_{2}$ & nand $_{3}$ \\
100 & 100 & \\
& 100 & 100 \\
010 & & 010 \\
\hline
\end{tabular}

The second line is in accordance with our original choice of control bits for nand $_{0}$ and nand $d_{1}$, but the third line seems to be a contradiction. However, we have to remember that all implied matches are real matches too. From Theorem 2 it follows that we can add inputs to a match and obtain an implied match. This means that we can just do a bitwise or on the bitvectors. This leads to the following 3 bit selector:

\begin{tabular}{|c|c|c|}
\hline nand $_{1}$ & nand $_{2}$ & nand $_{3}$ \\
110 & 100 & 110 \\
\hline
\end{tabular}

Or, graphically, to the selector configuration in Figure 6

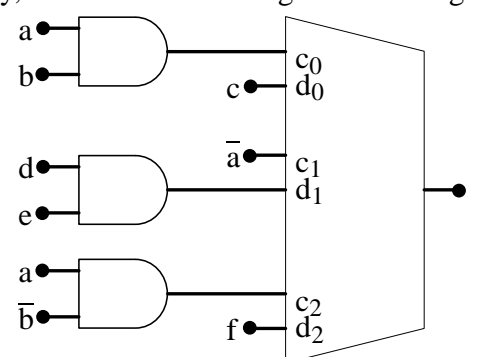

Figure 6: resulting selector circuit

From this we learn that we can add an input nand to a selector if the new input nand does have at least one match with all nands already in the selector. We say that the new nand is compatible with a selector if it has at least one match with all nands already in the selector.

To find all possible selectors for a candidate with $n$ input nands, we would have to try to grow selectors towards all possible $2^{n}$ subsets of input nands of the candidate. This becomes too large a number very quickly, although it might be feasible for candidates of 
smaller size. We have chosen to use the following heuristic: we assume the input nands are ordered. We then try to grow selectors starting from each input nand, adding input nands later in the ordering if they are compatible. If they are not compatible, we try the next one. In this way, for each candidate, we obtain a subset of all possible selectors.

\section{Checking completeness of selectors}

In the previous discussions we have only made sure that the control signals for the selector are orthogonal. But we have not worried about the possibility of them not being orthonormal. Yet, this might lead to undesirable high impedance output values in some selector implementations. Therefore, we check this condition. For every selector of every candidate we temporarily create the and gates to get the control input values for this selector. The output nets of these ands are not connected. Let $C$ be the set of control signals thus created. We then ask the Boolean Oracle what the value of All_O(C) is. If it is 1 , the control inputs of this selector can be 0 at the same time, and the selector is incomplete. If we decide to implement this selector, we will have to make it complete. If $A l l O(C)=0$, the selector is complete, and no further action is necessary.

We can make an incomplete selector complete by adding an extra bit to it. The data input of this bit is connected to the constant value 0 , and the control bit is the logic nor of the other control bits, which effectively selects this bit when none of the other bits are selected. As an example, the selector in Figure 6 is complete, because $a b+a \bar{b}+\bar{a}=1$.

\section{Merging selectors}

In many cases it is possible to merge selectors found in different candidates into one larger selector. In fact, the ability to do this has proved important to construct large selectors. In many cases logic synthesis, or other restructuring, will change a circuit such, that the algorithms described in this paper only find small selectors initially.

One type of merging we can perform is when a selector output is connected (perhaps inverted) to the data input of another selector. We call this input merging. This situation is pictured for a simple example in Figure 7.
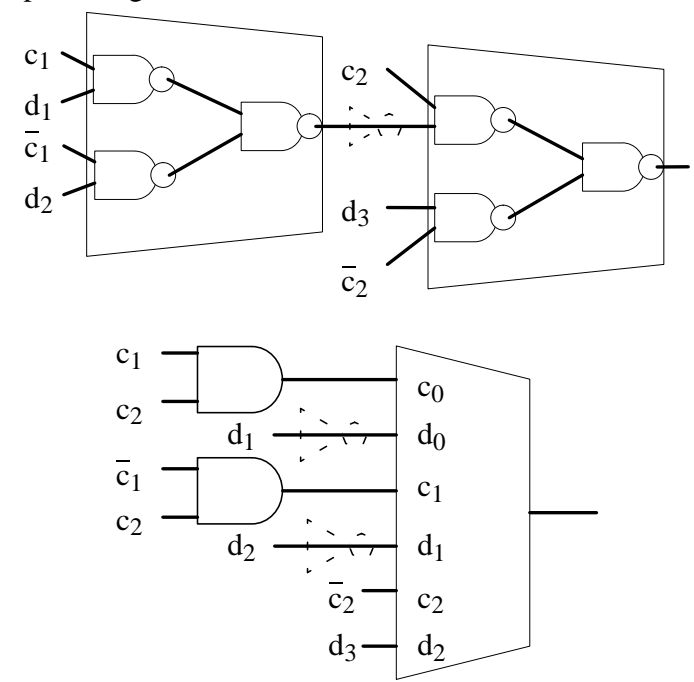

Figure 7: merging selectors: input merge

The second type of merging is pictured in Figure 8. In this case an input nand, which is part of a selector, is also an output nand of another selector. We call this, for obvious reasons, halfway merging. It is entirely equivalent to input merging with an inverter between the selectors.
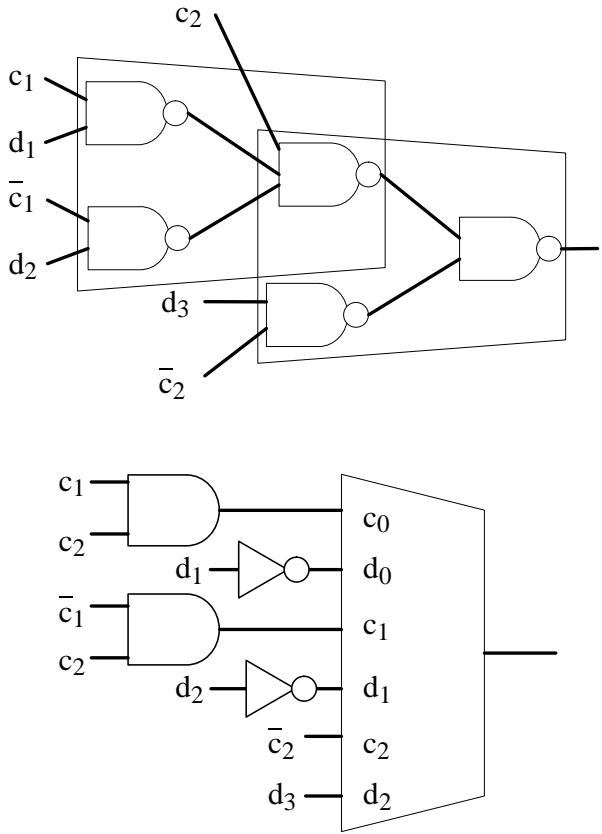

Figure 8: merging selectors: halfway merge

\section{Results}

The main results of this paper can be found in Table 2. It shows the selectors found in the entire set of MCNC multi-level combinatorial benchmark circuits ( 76 circuits). On the initial circuits we performed only some basic redundancy removal and a straight-forward conversion to nands. When we searched for selectors, we limited ourselves to candidates with at most 50 input nands. Each input nand was limited to 20 inputs. Both limitations assured us that we would have reasonable run times. The 6 circuits marked with ${ }^{2}$ ) hit these limits, and were not fully explored, but some selectors were found nonetheless. The 4 circuits marked with ${ }^{1}$ ) did run into the limits, and produced no selectors initially. On these examples we performed kernel factoring, after which they did not run into the limits any more and produced some selectors. In the benchmark circuits not listed in the table (C1355, C17, C432, C6288, cm138a, cm42a, decod, i3, i4, i5, majority, vda) we did not find any selectors.

Figure 9 summarizes the estimated saving in terms of the number of transistors for all benchmark circuits. It is obvious that quite a few circuits show considerable savings, up to $82 \%$.

Table 2 lists all the selectors found in the circuits. Please note that there are no columns for 9 and 11 input selectors, because these were not found in this test.

The table column labeled 'total sel bits' gives the sum of all selector bits in the previous columns, to give an indication of the total selector contribution. In a pass transistor implementation this would be the total number of transistors used in all selectors. The column 'trans. before 100\%' gives an indication of how many transistors the circuit used before the selectors were found. Because the circuits are transformed to nands only, this number is twice the total number of inputs to nands. This assumes a one-to-one mapping of nand expressions to nands from the library, which is of course not entirely accurate, but it is a good indication. The '100\%' indi- 


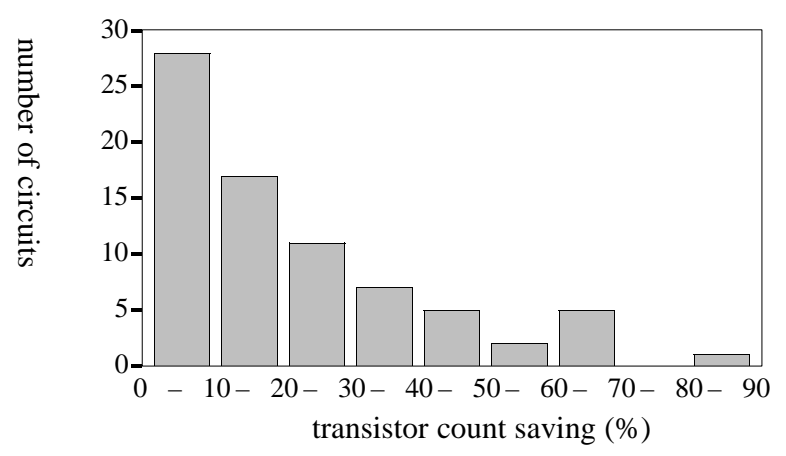

Figure 9: transistor count savings over all MCNC benchmark examples

cates that this number is used as a reference for other values. Column 'tr. after \%' gives the percentage of transistors in nands left after putting in the selectors and their surrounding logic (ands to and the data and control bits, nors to generate the control signal for the extra selector bits added to make incomplete selectors complete). Clearly, in many cases a substantial amount of the original logic is replaced by selectors. Column 'tr. sel \%' gives the number of transistors used in the selectors and the and-logic for the control and data bits. We assume this and-logic is also performed by serial pass transistors to the selectors. The quantity is expressed as a percentage of the original number of transistors. Column 'tr. nors \%' gives the amount of transistors needed for the nors. Again we take twice the number of inputs to the nors here. This amount too is expressed as a percentage of the original transistor count. It is an indication of how much extra logic was necessary to make incomplete selectors complete. As you can see, in most cases this number is very small. Column 'total \%', adds the previous three columns, to show the total \% of transistors left after replacing nands by selectors.

Figure 10 summarizes the number of selectors of different sizes

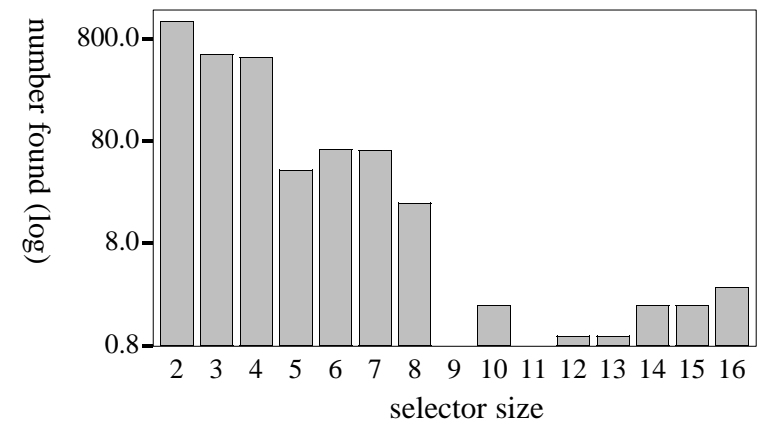

Figure 10: number of selectors of a specific size found in all MCNC benchmark examples

found in all benchmark examples combined. It clearly shows that many selectors were found, most of which were 8 bits wide or less. The final column of table 2 shows how much CPU time (in seconds on an IBM RS6000/370, a 60 MIPS machine) was spent to find and construct the selectors.

\section{Conclusions}

The theory and algorithms presented in this paper allow automatic mapping to selectors based on Boolean properties of signals in the circuit. Although the algorithms have an exponential worst case run time complexity, for practical circuits they are fast enough. We have shown that there is a large potential to area savings when pass transistor selectors would be used in the MCNC benchmark circuits.

\section{Acknowledgements}

The authors want to thank Dan Brand, Robert Damiano and Louise Trevillyan for many discussions and helpful hints during the implementation of the algorithms described in the paper in the IBM BooleDozer logic synthesis system.

\section{References}

[DET87] Detjens, E, G. Gannot, R. Rudell, A. SangioVANNI-VINCENTELLI and A. WANG, "Technology Mapping in MIS", Proceedings of the International Conference on Computer-Aided Design 1987, pp. 116-119.

[KUN90]: Kunda, R.P., P. NARAin, J.A. AbRaham and B.D RATHI, "Speed up of Test Generation using High Level Primitives", Proceedings of the 27th Design Automation Conference, June 1990, pp. 594-599.

[KUN92]: KUNDU, S., L.H. HuISMAN, I. NAIR, V.S. IYENGAR and L.N. REDDY, "A small Test Generator for Large Designs", Proceedings of the International Test Conference, Sept. 1992, pp. 30-40.

[LAN93]: LAN, S.H., R. GopisetTy and K.R. DhaRmarajan, "CMAP - Technology Mapping for Multiplexor Based FPGA Architectures Using Certificate of Generic Boolean Function", Proceedings of the IEEE 1993 Custom Integrated Circuits Conference, pp 3.4.1 - 3.4.4.

[MAI90] MaILhot, F. and G. DE Micheli, "Technology Mapping using Boolean Matching and Don't Care Sets", Proceedings of the European Design Automation Conference 1990, pp. 212-216.

[RUD93]: RUDELL, R., "Dynamic Variable Ordering for Ordered Binary Decision Diagrams", Workshop Notes of the International Workshop on Logic Synthesis 1993, May 1993, pp 3a-1 - 3a-12

[WHI83]: WhitaKer, S., "Pass-transistor networks optimize n-MOS logic", Electronics, September 22, 1983, pp. 144-148.

[ZHU93]: ZHU, K. and D.F. WonG, "Fast Boolean Matching for Field-Programmable Gate Arrays", Proceedings of the 1993 European Design Automation Conference (EuroDAC), pp 352-357. 
Table 2: Results on all MCNC multi-level benchmark circuits.

\begin{tabular}{|c|c|c|c|c|c|c|c|c|c|c|c|c|c|c|c|c|c|c|c|c|}
\hline \multirow[t]{2}{*}{ name } & \multicolumn{13}{|c|}{ number of selectors of size } & \multirow{2}{*}{\begin{tabular}{|l} 
total \\
sel \\
bits
\end{tabular}} & \multirow{2}{*}{$\begin{array}{l}\text { trans. } \\
\text { before } \\
100 \%\end{array}$} & \multirow{2}{*}{ 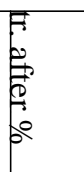 } & $\vec{F}$ & $\stackrel{F}{F}$ & $\overrightarrow{0}$ & 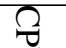 \\
\hline & 2 & 3 & 4 & 5 & 6 & 7 & 8 & \begin{tabular}{|l|}
1 \\
0
\end{tabular} & $\begin{array}{l}1 \\
2\end{array}$ & $\begin{array}{l}1 \\
3\end{array}$ & $\begin{array}{l}1 \\
4\end{array}$ & $\begin{array}{l}1 \\
5\end{array}$ & $\begin{array}{l}1 \\
6\end{array}$ & & & & & $a^{9}$ & & en \\
\hline 9symml & 14 & 2 & & & & & & & & & & & & 31 & 750 & 77 & 6 & 1 & 84 & 2.6 \\
\hline C1908 & 38 & & & & & & 1 & & & & & & & 84 & 1802 & 68 & 7 & 1 & 76 & 6.2 \\
\hline$\left(2670^{2}\right)$ & 29 & 2 & 25 & & & & & & & & & & & 164 & 2440 & 56 & 8 & 0.2 & 76 & 14 \\
\hline C3540 & 17 & 17 & 13 & 1 & 6 & 4 & 4 & & & & 2 & 1 & 1 & 297 & 3948 & 63 & 11 & 1 & 75 & 27 \\
\hline C499 & 48 & & & & & & & & & & & & & 96 & 1856 & 69 & 5 & 0 & 74 & 4.4 \\
\hline C5315 & 77 & 15 & 78 & 3 & 3 & & & & & & & & & 526 & 6482 & 45 & 10 & 0.5 & 56 & 23 \\
\hline C7552 & 158 & 2 & 23 & 8 & & & & & & & & & & 454 & 8266 & 66 & 7 & 0.9 & 74 & 45 \\
\hline C880 & 11 & 8 & & & & & & & & & & & & 46 & 1336 & 88 & 13 & 3 & 94 & 4.6 \\
\hline alu $\left.2^{2}\right)$ & 2 & 3 & & 3 & 1 & 1 & 2 & 1 & 1 & & & & & 79 & 1360 & 61 & 12 & 8 & 81 & 17 \\
\hline alu $4^{2}$ ) & 2 & 4 & 1 & 3 & 5 & 3 & & 1 & & 1 & & 1 & 1 & 140 & 2726 & 61 & 12 & 8 & 81 & 50 \\
\hline apex6 & 15 & 67 & & 5 & 11 & & & & & & & & & 322 & 2674 & 39 & 14 & 5 & 58 & 8.9 \\
\hline apex7 & 7 & 10 & & & & 1 & & & & & & & & 51 & 902 & 71 & 9 & 5 & 85 & 3.1 \\
\hline b1 & 2 & & & & & & & & & & & & 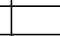 & 4 & 46 & 57 & 9 & 0 & 66 & 0.6 \\
\hline b9 & 4 & & & & & & & & & & & & & 8 & 500 & 91 & 2 & 0 & 93 & 1.6 \\
\hline $\mathrm{c} 8$ & 15 & 7 & 1 & & & & & & & & & & & 55 & 710 & 43 & 17 & 3 & 63 & 3.7 \\
\hline $\mathrm{cc}$ & & 1 & & & & & & & & & & & & 3 & 312 & 97 & 1 & 1 & 99 & 1.1 \\
\hline cht & 35 & 1 & & & & & & & & & & & & 73 & 678 & 16 & 20 & 0 & 36 & 2.8 \\
\hline $\mathrm{cm} 150 \mathrm{a}$ & & & & & & & & & & & & & 1 & 16 & 198 & 7 & 26 & 0 & 33 & 1.3 \\
\hline $\mathrm{cm} 151 \mathrm{a}$ & & & & & & & 1 & & & & & & & 8 & 100 & 32 & 20 & 0 & 52 & 0.9 \\
\hline $\mathrm{cm} 152 \mathrm{a}$ & & & & & & & 1 & & & & & & & 8 & 86 & 7 & 28 & 0 & 35 & 1.2 \\
\hline $\mathrm{cm} 162 \mathrm{a}$ & 4 & & & & & & & & & & & & & 8 & 174 & 82 & 5 & 0 & 87 & 1.0 \\
\hline cm163a & 4 & & & & & & & & & & & & & 8 & 172 & 81 & 5 & 0 & 86 & 0.9 \\
\hline $\mathrm{cm} 82 \mathrm{a}$ & 2 & & & & & & & & & & & & & 4 & 100 & 76 & 4 & 0 & 80 & 0.6 \\
\hline $\mathrm{cm} 85 \mathrm{a}$ & 4 & & & & & & & & & & & & & 8 & 198 & 80 & 4 & 0 & 84 & 1.0 \\
\hline $\mathrm{cmb}$ & 3 & & & & & & & & & & & & & 6 & 168 & 79 & 6 & 0 & 85 & 0.9 \\
\hline comp & & & 1 & & & & & & & & & & & 4 & 368 & 95 & 2 & 1 & 98 & 1.4 \\
\hline cordic & 8 & & & & & & & & & & & & & 16 & 358 & 75 & 4 & 0 & 79 & 1.7 \\
\hline count & & 16 & & & & & & & & & & & & 48 & 544 & 47 & 15 & 0 & 62 & 2.2 \\
\hline $\mathrm{cu}$ & 1 & & 1 & & & & & & & & & & & 6 & 224 & 84 & 4 & 0 & 88 & 1.0 \\
\hline dalu & 69 & 47 & & & & & & & & & & & & 279 & 4278 & 61 & 9 & 3 & 73 & 17 \\
\hline $\left.\operatorname{des}^{2}\right)$ & 143 & 67 & 36 & 4 & 11 & 55 & 10 & & & & & & & 1182 & 15144 & 35 & 12 & 3 & 50 & 124 \\
\hline example2 & 21 & 7 & 8 & 1 & & & & & & & & & & 100 & 1098 & 52 & 11 & 5 & 68 & 4.7 \\
\hline $\mathrm{f} 51 \mathrm{~m}$ & 2 & 1 & 4 & & & & & & & & & & & 23 & 788 & 77 & 7 & 0 & 84 & 13 \\
\hline frg $\left.1^{1}\right)$ & 1 & & & & & & & & & & & & & 2 & 556 & 98 & 0.4 & 0 & 98 & 1.9 \\
\hline frg2 & 50 & 79 & 1 & & & & & & & & & & & 341 & 4622 & 60 & 13 & 1 & 74 & 40 \\
\hline i10 & 152 & 27 & 9 & 10 & 1 & 1 & & & & & & & & 484 & 8218 & 66 & 8 & 2 & 76 & 28 \\
\hline i1 & & 2 & & & & & & & & & & & & 6 & 168 & 85 & 5 & 5 & 95 & 0.9 \\
\hline$\left.i^{2}{ }^{2}\right)$ & 1 & & & & & & & & & & & & & 2 & 876 & 99 & 0.3 & 0 & 99 & 3.4 \\
\hline i6 & 1 & & 66 & & & & & & & & & & & 266 & 1752 & 22 & 20 & 0.3 & 43 & 7.7 \\
\hline i7 & 2 & & 64 & & & & & & & & & & & 260 & 2188 & 19 & 15 & 0 & 34 & 11 \\
\hline i8 & & 23 & 64 & 1 & 32 & & & & & & & & & 522 & 5538 & 45 & 11 & 0.5 & 57 & 39 \\
\hline i9 & & 8 & 56 & & & & & & & & & & & 248 & 1954 & 33 & 13 & 0.7 & 47 & 9.1 \\
\hline $\left.\mathrm{k} 2^{1}\right)$ & 1 & 16 & 2 & & & & 1 & & & & & & & 62 & 3104 & 89 & 3 & 3 & 95 & 8.5 \\
\hline lal & 1 & 1 & & & & & & & & & & & & 5 & 612 & 95 & 1 & 1 & 97 & 1.8 \\
\hline mux & & & & & & & & & & & & & 1 & 16 & 198 & 7 & 26 & 0 & 33 & 1.5 \\
\hline my_adder & & & 16 & & & & & & & & & & & 64 & 930 & 48 & 12 & 0 & 60 & 3.8 \\
\hline pair & 95 & 44 & 2 & & & & & & & & & & & 330 & 5812 & 70 & 7 & 0.6 & 78 & 22 \\
\hline parity & 1 & & & & & & & & & & & & & 2 & 240 & 95 & 1 & 0 & 96 & 0.8 \\
\hline pcle & & 1 & 7 & & & & & & & & & & & 31 & 238 & 30 & 19 & 19 & 68 & 1.3 \\
\hline pcler8 & & 1 & 7 & & & & & & & & & & & 31 & 302 & 56 & 15 & 15 & 86 & 1.4 \\
\hline $\mathrm{pm} 1$ & & 1 & & & & & & & & & & & & 3 & 246 & 92 & 3 & 2 & 97 & 1.0 \\
\hline rot & 18 & 14 & 2 & 2 & 2 & & & & & & & & & 96 & 3372 & 80 & 6 & 1 & 87 & 84 \\
\hline sct & 3 & & & & & & & & & & & & & 6 & 502 & 90 & 3 & 0 & 93 & 1.6 \\
\hline tcon & 8 & & & & & & & & & & & & & 16 & 98 & 2 & 16 & 0 & 18 & 0.9 \\
\hline term1 & 5 & 10 & 3 & & & & & & & & & & & 52 & 1422 & 83 & 7 & 3 & 93 & 5.5 \\
\hline $\left.\mathrm{t} 481^{\mathrm{I}}\right)$ & 1 & 1 & 1 & 1 & & & & & & & & & & 14 & 294 & 76 & 7 & 6 & 89 & 2.6 \\
\hline too large ${ }^{1}$ ) & 3 & 8 & & & & & & & & & & & & 30 & 2402 & 94 & 2 & 1 & 97 & 6.4 \\
\hline $\mathrm{ttt} 2$ & 17 & 5 & & & & & & & & & & & & 49 & 1130 & 71 & 10 & 2 & 83 & 4.7 \\
\hline unreg & 32 & & & & & & & & & & & & & 64 & 456 & 44 & 21 & 0 & 65 & 2.1 \\
\hline$\left.x 1^{2}\right)$ & 8 & 2 & & & & & & & & & & & & 22 & 4326 & 95 & 2 & 0.2 & 97 & 447 \\
\hline $\mathrm{x} 2$ & 1 & 1 & & & & & & & & & & & & 5 & 166 & 70 & 11 & 0 & 81 & 0.9 \\
\hline $\mathrm{x} 3$ & 46 & 32 & 16 & & & & & & & & & & & 252 & 4386 & 73 & 8 & 2 & 83 & 15 \\
\hline $\mathrm{x} 4$ & 9 & 13 & 20 & & & & & & & & & & & 137 & 2020 & 58 & 10 & 2 & 70 & 8.1 \\
\hline $\mathrm{z} 4 \mathrm{ml}$ & & 2 & 1 & & & & & & & & & & & 10 & 628 & 87 & 4 & 0 & 91 & 13 \\
\hline total & 1191 & 568 & 528 & 42 & 67 & 65 & 20 & 2 & 1 & 1 & 2 & 2 & 3 & 7572 & & & & & 75 & \\
\hline
\end{tabular}

1) No selectors initially, only after kernel factoring

2) Not fully explored, fanin limit exceeded 